Suspicion of smallpox should be communicated immediately to the medical officer for environmental health, whose responsibility it is to put into operation control measures. Clearly the patient with suspected or proved smallpox must be isolated; the closer the primary contact the greater the need for vaccination and without delay-this is usually offered to contacts of close contacts also. Close contacts not vaccinated within three days of exposure may be offered immunoglobulin and methisazone after vaccination is carried out, and all close contacts should be under surveillance daily for 16 days after the last possible exposure. Pregnant women and small children with eczema should also be given immunoglobulin at the time of vaccination. Rooms should be disinfected and clothes and bedding disinfected or burnt. It is wise for all those medical, nursing, and paramedical staff who might be exposed to a smallpox patient to keep up to date with vaccination.

Plans for control have been carefully worked out on the basis of the observations cited earlier; mass vaccination should not be carried out, and all control measures should be left to the medical officer for environmental health, who should be kept fully informed of all happenings. The Memorandum on the Control of Outbreaks of Smallpox recently published by the Department of Health and Social Security and the Welsh Office gives details of the way in which such control measures will be put into effect.

\section{Plasticity in the nervous system}

Among the laws handed down to each medical student from the Mount Sinai of his early education is the ruling that the central nervous system is incapable of structural change. The capacity of a tissue to produce an alteration in a pattern of pre-existing connections may be called its plasticity. During fetal development the nervous system displays continuous plasticity, when its pattern of connections is altered either by the birth of new cells or by the growth and ramification of previously existing axonal and dendritic processes. Why should this property suddenly fall to nothing during extrauterine life? If plasticity were, after all, preserved into adulthood, clinicians should be able to use it. For example, in principle might the mammalian nervous system be able to reconstitute its pattern after injury?

Evidence in support of the preservation of plasticity after birth has mostly come to light during the last six years, and the subject was reviewed and updated last month at a meeting of the Royal Society. C Blakemore, from Cambridge, summarised the evidence that the visual projection to the cortex at birth is not fixed but remains subject to the modifying influence of environmental traffic during a critical period, which in the cat is at its most sensitive at about 6 weeks of age. The cortical neurons are arranged in columns, each of which at birth is characterised by a preset level of preference for being driven by one eye or the other. Nevertheless, during the critical period (beginning at the time the eyes open) this preference pattern can be varied by visual experience: the cortical neurons of an animal in which one eye has been temporarily shut by suturing the eyelids display a definite preference for being driven by the non-deprived eye. If deprivation is carried onfor long enough the number of cortical terminals normally relayed from the deprived eye decreases. This suggests a functional alteration followed by a structural one. Furthermore, if an animal is subjected to "environmental surgery" by rearing it in a cage with striped walls either of predominantly vertical or horizontal pattern then the number of cortical neurons capable of responding to the missing kind of stripe decreases during the period of deprivation. This physiological change is accompanied by a structural realignment of dendritic branching in those cells thought to respond with orientation preference.

When we come to the central nervous systems of adults there is still evidence that terminals, given the appropriate stimulus, can change their pattern. G Raisman's work on the septal nucleus of the rat has shown that when the septal nucleus is deprived of one of its two main afferent paths fibre terminals from the other grow in so as to occupy the vacated synaptic regions. Perhaps the most surprising feature of this phenomenon is that it occurs at all. There is as yet no evidence of the Blakemore type of a controlling functional interaction with the external environment, and the rewoven pattern of connections may leave the animal worse off than before. As Raisman pointed out, collateral innervation of this type may even serve to inhibit the restitution of the original pattern, which perhaps could otherwise proceed by fibre regeneration.

Evidence that the lower vertebrates may display even more extensive plastic readjustments was summarised by $\mathrm{T} J$ Horder from Oxford and M J Keating from Mill Hill. Fibres in the optic tracts of frogs or fishes are capable of true regeneration. When allowed to grow back into the target optic centre, half of which has been removed, these fibres grow at first towards the place on the target (the tectum) where each would have terminated if the whole target had been there. As time goes on and more fibres regenerate into the tectum, however, fibres redistribute themselves, so that eventually the whole fibre population ends in an ordered fashion in the half-tectum, thus providing most fibres with terminal sites that they would not normally have encountered. One method of accounting for such findings, and for the plasticity of the intertectal connections that are probably responsible for the frog's binocular vision, is to think that fibres interact with one another, signalling such messages as the amount of terminal space available and perhaps some details of their own sites of origin on the retina.

These data may be brought together by conceiving the development of a nervous subsystem as divided into three phases. The first phase is characterised by fibre growth and the induction of dendritic arborisation patterns. In the second phase interaction with the animal's environment can tune the pattern of connections formed into its maximum efficiency. In the last phase, dominated by the maintenance of stable connections rather than the formation of fresh ones, residual capacity for fibre regeneration becomes attenuated and elicitable phenomena become restricted to collateral reinnervation from nearby terminals.

The message for the clinician is thus still a fairly sombre one. In the foreseeable future possibly some use will be made of the increasing body of evidence about critical periods. (More effective treatment of strabismus, for example, would be made possible by early diagnosis combined with exact knowledge of the time at which treatment could safely be stopped. At the moment treatment is probably left too late to catch any substantial fraction of the critical period.) Nevertheless, the restitution of connection patterns after large-scale trauma to the central nervous system must await the development of new techniques, either of enhancing true fibre regeneration or of manipulating functionally apposite terminal rearrangements of the critical period type. As yet we are a very long way from achieving either of these objectives. 